\title{
Bridging shear transformation zone to the atomic structure of amorphous solids
}

\author{
M. Zhang, Y.J. Wang, L.H. Dai* \\ State Key Laboratory of Nonlinear Mechanics, Institute of Mechanics, Chinese Academy of Sciences, Beijing 100190, China
}

\section{A R T I C L E I N F O}

\section{Article history:}

Received 21 October 2014

Received in revised form 13 December 2014

Accepted 16 December 2014

Available online 20 December 2014

\section{Keywords:}

Amorphous alloys;

Shear transformation zone;

Amorphous structure;

Mechanical behavior

\begin{abstract}
A B S T R A C T
To correlate the atomic structure and mechanical behavior of amorphous solids, bridging shear transformation zone (STZ) to the atomic structure is crucial. Referring to dislocation of crystals and according to the concept of shear transformation in amorphous solids, from a mean perspective, an atomic structure-based shear transformation zone (SSTZ) based on radial distribution function (RDF) where an average atom is surrounded by atoms of different shells, is proposed. The elementary deformation event of the disordered structure is considered as a mean atomic rearrangement, i.e., a concordant rearrangement process between the atom shells in the SSTZ. The SSTZ will introduce no necessary modification into the transition state theory-based flow equation of amorphous alloys and provide both the universal yielding criterion and the anelastic deformation of amorphous alloys with a structure basis in terms of the shift of the peak on RDF under loading. Besides, regarding with issues like shear dilation and Poisson ratio etc., profound understandings can be achieved for the clear structural characteristic of the SSTZ.
\end{abstract}

(c) 2014 Elsevier B.V. All rights reserved.

\section{Introduction}

The elementary deformation unit, or more exactly deformation event, of disordered structure is of great importance to elucidate the mechanical behaviors of amorphous solids on the atomic structure level [1-3]. The effort to clarify such a unit can find its trail dating back to the 1930s [4-6], i.e., the concept of dislocation [7,8], a line defect defined on the crystal lattice, of which the glide on the lattice is the primary deformation mechanism of crystals [9]. Coming to amorphous solids, a concept of shear transformation in local atomic configuration, lately named shear transformation zone (STZ), is proposed by analogy to dislocations $[10,11]$. Although dislocation is undoubtedly observed with modern microscopy technology and the related theory on the various deformation behaviors of crystals is developed, the STZ in amorphous solids is still obscure and confusing for the isotropic and homogeneous contrast of the image of amorphous solids under high resolution electron microscopy [3,12-14]. However, many issues in the deformation of amorphous solids, like yielding and shear dilation, reckon on the structural characteristic of STZ [10]. What makes the situation more confusing, widespread consensus has been reached that STZ seems "event-oriented" rather than an entity of a specific structure like the clearly observed dislocation of crystals [2]. Computer simulations have also indicated that the size of STZ "events" follows a power law distribution and exhibits no characteristic size [15]. This dilemma

\footnotetext{
* Corresponding author.

E-mail address: lhdai@lnm.imech.ac.cn (L.H. Dai).
}

of STZ stands as an unavoidable obstacle in correlating the mechanical behavior of amorphous solids to their atomic structure, especially for the clear structure variation in deformation observed in recent studies $[16,17]$.

Besides electron microscopy, examination on amorphous structure is carried out mainly with synchrotron X-ray scattering tests [18-20], by which the atom packing characteristic can be obtained from the scattering pattern in the form of structure factor in the reciprocal space or radial (or pair) distribution function (RDF or PDF) in the real space. With enhanced X-ray intensity, scattering tests with in-situ loading circumstance in which the shift of the RDF or structure factor can be measured showing the length scale dependent atomic strain have been performed and provide important structure messages of amorphous alloys in deformation [21-23] whereas, the length scale dependent atomic strain obtained in scattering tests is averaged over the part of sample that participates in scattering the incident X-ray beam and individual STZs cannot be resolved. Therefore, in spite that the concept of STZ observed in the rearrangements of amorphous systems, like foam-craft model [24] and colloids [25], presents an important picture of the deformation of amorphous solids, the structural characteristic of STZ remains vague with nowadays structure characterization technique in understanding the mechanical behaviors of amorphous solids.

In this work, to make the best of the structure messages from in-situ loading scattering tests in understanding the mechanical behavior of amorphous solids, referring to dislocation of crystals and according to the concept of shear transformation, from a mean perspective, an atomic structure-based STZ (SSTZ) is proposed based on RDF to make up the 
missing structural characteristic of STZ in Part 2. The concept of SSTZ is justified in Part 3, and the applicability and application of the SSTZ are discussed in Part 4. The main conclusions are summarized in Part 5.

\section{Method}

Looking into the works on dislocation, it is important to note that the elementary deformation $\psi$ of crystals induced by the glide [7] of mobile dislocations is calculated as [26]: $\psi=b \rho_{m} / \sqrt{\rho_{f}}$, where $\rho_{m}$ is the mobile dislocation density (the length of the mobile dislocation line in unit volume); $\rho_{f}$ is the forest dislocation density; $b$ is the modulus of the Burgers vector of dislocation [9]. However, the glide of a dislocation, similar to the operation of a STZ surrounded by its matrix in amorphous solids, will not change the shape of the grain until it reaches the grain boundary. Hence, understanding the strain of dislocation glide from the perspective of individual dislocation glide [4] seems inappropriate and so does understanding the strain induced by the operation of an individual STZ in amorphous solids. Noting the coefficient $\rho_{m} / \sqrt{\rho_{f}}$, where $1 / \sqrt{\rho_{f}}$ is the mean space between the forest dislocations in a glide plane which act as obstacles to resist the glide of the mobile dislocations, it means that the macroscopic plastic deformation of crystals is actually calculated as a mean dislocation gliding along its Burgers vector in the glide plane, i.e., a mean dislocation of $\rho_{m}$ in length and of a Burgers vector of modulus $b$ sweeps through an equivalent crystal grain of a size of $\sqrt{\rho_{f}} / \rho_{m}$ in the dislocation glide direction and induces a macroscopic strain of $\psi$. From this aspect of view, in amorphous solids, it would also be more appropriate to understand the deformation mechanism from a similar mean perspective to resolve the effect of the surrounding matrix in the operation of individual STZs. For the long range disorder, the mean atomic structure of amorphous solids is characterized with radial distribution function (RDF) $g(r)$ schematically illustrated in Fig. 1, where different shells of atoms around the average atom are shown, noting that the splitting of the second strong peak is not drawn for clarity. In the following part, we will justify the STZ based on RDF from the mean perspective with a structure basis, i.e., an atomic structure-based STZ (SSTZ), within the state of the art of researches on amorphous alloys.

\section{Results}

Firstly, we have calculated the average atom size of a series of amorphous alloys [27] that have been fabricated, which turns out to be of a radii of $0.14-0.2 \mathrm{~nm}$ as shown in Table 1 . Meantime, it is important to note that the scale range of the RDF is 0 to $1.5-2 \mathrm{~nm}[18,28]$, a domain of about 10-atom size comparing to the average atom radii. Based on these results, the number of atoms in the range of $0-2 \mathrm{~nm}$ on the RDF is roughly 1000 atoms, encompassing the size (1-3 nm in diameter) and the number of atoms (100-500) of a nowadays recognized STZ quite well $[29,30]$. Secondly, it has been reported that the length scale

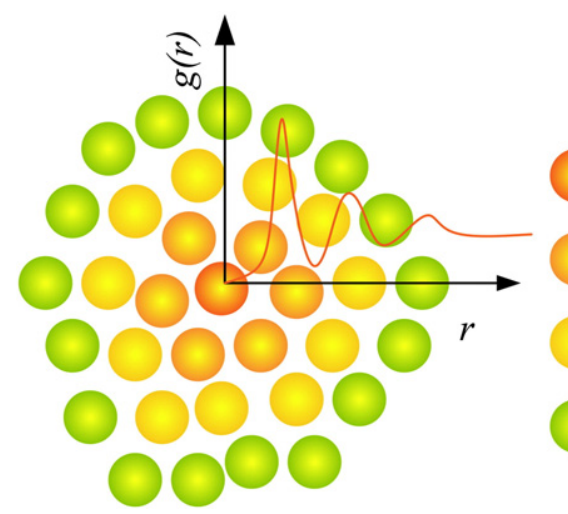

Average atom

1st shell

2nd shell

3rd shell
Table 1

Average atomic radii of amorphous alloys.

\begin{tabular}{|c|c|}
\hline Composition (at.\%) & Average atomic radii $(\AA)$ \\
\hline $\mathrm{Zr}_{62} \mathrm{Cu}_{15.4} \mathrm{Ni}_{12.6} \mathrm{Al}_{10}$ & 1.67 \\
\hline $\mathrm{Zr}_{59} \mathrm{Ta}_{5} \mathrm{Cu}_{18} \mathrm{Ni}_{8} \mathrm{Al}_{10}$ & 1.67 \\
\hline $\mathrm{Zr}_{41.2} \mathrm{Ti}_{13.8} \mathrm{Cu}_{12.5} \mathrm{Ni}_{10} \mathrm{Be}_{22.5}$ & 1.58 \\
\hline $\mathrm{Cu}_{47.5} \mathrm{Zr}_{47.5} \mathrm{Al}_{5}$ & 1.61 \\
\hline $\mathrm{Cu}_{60} \mathrm{Zr}_{20} \mathrm{Hf}_{10} \mathrm{Ti}_{10}$ & 1.56 \\
\hline$\left(\mathrm{Ti}_{0.5} \mathrm{Cu}_{0.5}\right)_{84} \mathrm{Ni}_{7} \mathrm{Hf}_{5} \mathrm{Zr}_{3} \mathrm{Si}_{1}$ & 1.54 \\
\hline $\mathrm{Ni}_{62.5} \mathrm{Zr}_{20} \mathrm{Nb}_{15} \mathrm{Pd}_{2.5}$ & 1.52 \\
\hline $\mathrm{Ni}_{60} \mathrm{Zr}_{20} \mathrm{Nb}_{15} \mathrm{Pd}_{5}$ & 1.52 \\
\hline $\mathrm{Ni}_{57.5} \mathrm{Zr}_{20} \mathrm{Nb}_{15} \mathrm{Pd}_{7.5}$ & 1.52 \\
\hline $\mathrm{Ni}_{55} \mathrm{Zr}_{20} \mathrm{Nb}_{15} \mathrm{Pd}_{10}$ & 1.53 \\
\hline $\mathrm{Ni}_{52.5} \mathrm{Zr}_{20} \mathrm{Nb}_{15} \mathrm{Pd}_{12.5}$ & 1.53 \\
\hline$\left[\left(\mathrm{Fe}_{0.8} \mathrm{Co}_{0.1} \mathrm{Ni}_{0.1}\right)_{0.75} \mathrm{~B}_{0.2} \mathrm{Si}_{0.05}\right]_{96} \mathrm{Nb}_{4}$ & 1.40 \\
\hline$\left[\left(\mathrm{Fe}_{0.6} \mathrm{Ni}_{0.4}\right)_{0.75} \mathrm{~B}_{0.2} \mathrm{Si}_{0.05}\right]_{96} \mathrm{Nb}_{4}$ & 1.40 \\
\hline $\mathrm{Fe}_{76} \mathrm{Si}_{9.6} \mathrm{~B}_{8.4} \mathrm{P}_{6}$ & 1.47 \\
\hline$\left(\mathrm{Fe}_{0.76} \mathrm{Si}_{0.096} \mathrm{~B}_{0.084} \mathrm{P}_{0.06}\right)_{99.9} \mathrm{Cu}_{0.1}$ & 1.47 \\
\hline $\mathrm{Zr}_{65} \mathrm{Cu}_{10} \mathrm{Ni}_{10} \mathrm{Al}_{10}$ & 1.67 \\
\hline $\mathrm{Zr}_{64.13} \mathrm{Cu}_{15.75} \mathrm{Ni}_{10.12} \mathrm{Al}_{10}$ & 1.67 \\
\hline $\mathrm{Zr}_{61.88} \mathrm{Cu}_{18} \mathrm{Ni}_{10.12} \mathrm{Al}_{10}$ & 1.66 \\
\hline $\mathrm{Zr}_{55} \mathrm{Cu}_{7} \mathrm{Co}_{19} \mathrm{Al}_{19}$ & 1.66 \\
\hline $\mathrm{Zr}_{57} \mathrm{Cu}_{15.4} \mathrm{Nb}_{5} \mathrm{Al}_{10} \mathrm{Ni}_{12.6}$ & 1.66 \\
\hline $\mathrm{Zr}_{57} \mathrm{Cu}_{20} \mathrm{Ti}_{5} \mathrm{Al}_{10} \mathrm{Ni}_{8}$ & 1.66 \\
\hline $\mathrm{Au}_{49} \mathrm{Ag}_{5.5} \mathrm{Pd}_{2.3} \mathrm{Cu}_{26.9} \mathrm{Si}_{16.3}$ & 1.63 \\
\hline$\left(\mathrm{Zr}_{0.59} \mathrm{Cu}_{0.22} \mathrm{Ti}_{0.06} \mathrm{Ni}_{0.13}\right)_{85.7} \mathrm{Al}_{14.3}$ & 1.62 \\
\hline $\mathrm{Zr}_{45} \mathrm{Cu}_{45} \mathrm{Gd}_{3} \mathrm{Al}_{7}$ & 1.62 \\
\hline $\mathrm{Au}_{55} \mathrm{Cu}_{25} \mathrm{Si}_{20}$ & 1.62 \\
\hline $\mathrm{Zr}_{54} \mathrm{Cu}_{46}$ & 1.60 \\
\hline $\mathrm{Zr}_{46.75} \mathrm{Ti}_{8.25} \mathrm{Cu}_{10.15} \mathrm{Ni}_{10} \mathrm{Be}_{27.25}$ & 1.59 \\
\hline $\mathrm{Zr}_{41} \mathrm{Ti}_{14} \mathrm{Cu}_{12.5} \mathrm{Ni}_{10} \mathrm{Be}_{22.5}$ & 1.57 \\
\hline $\mathrm{Zr}_{48} \mathrm{Fe}_{8} \mathrm{Cu}_{12} \mathrm{Nb}_{8} \mathrm{Be}_{24}$ & 1.59 \\
\hline $\mathrm{Ni}_{45} \mathrm{Zr}_{25} \mathrm{Ti}_{20} \mathrm{Al}_{10}$ & 1.56 \\
\hline $\mathrm{Pd}_{77.5} \mathrm{Cu}_{6} \mathrm{Si}_{16.5}$ & 1.56 \\
\hline $\mathrm{Pd}_{60} \mathrm{Cu}_{20} \mathrm{P}_{20}$ & 1.51 \\
\hline $\mathrm{Pd}_{40} \mathrm{Cu}_{40} \mathrm{P}_{20}$ & 1.50 \\
\hline $\mathrm{Pd}_{39} \mathrm{Ni}_{10} \mathrm{Cu}_{30} \mathrm{P}_{21}$ & 1.47 \\
\hline $\mathrm{Fe}_{53} \mathrm{Cr}_{15} \mathrm{Mo}_{14} \mathrm{Er}_{1} \mathrm{C}_{15} \mathrm{~B}_{6}$ & 1.47 \\
\hline $\mathrm{Fe}_{74.5} \mathrm{Mo}_{5.5} \mathrm{P}_{12.5} \mathrm{C}_{5} \mathrm{~B}_{2.5}$ & 1.47 \\
\hline $\mathrm{Fe}_{70} \mathrm{Mo}_{5} \mathrm{Ni}_{5} \mathrm{P}_{12.5} \mathrm{C}_{5} \mathrm{~B}_{2.5}$ & 1.40 \\
\hline $\mathrm{Fe}_{61} \mathrm{Mn}_{10} \mathrm{Cr}_{4} \mathrm{Mo}_{6} \mathrm{Er}_{1} \mathrm{C}_{15} \mathrm{~B}_{6}$ & 1.40 \\
\hline $\mathrm{Pd}_{64} \mathrm{Ni}_{16} \mathrm{P}_{20}$ & 1.44 \\
\hline $\mathrm{Pt}_{57.5} \mathrm{Ni}_{5} \mathrm{Cu}_{14.7} \mathrm{P}_{22.8}$ & 1.49 \\
\hline $\mathrm{Pt}_{60} \mathrm{Ni}_{15} \mathrm{P}_{25}$ & 1.50 \\
\hline $\mathrm{Ni}_{60} \mathrm{Nb}_{35} \mathrm{Sn}_{5}$ & 1.50 \\
\hline $\mathrm{Ni}_{60}\left(\mathrm{Nb}_{0.8} \mathrm{Ta}_{0.2}\right)_{34} \mathrm{Sn}_{6}$ & 1.50 \\
\hline $\mathrm{Zr}_{50} \mathrm{Cu}_{50}$ & 1.51 \\
\hline $\mathrm{Zr}_{48} \mathrm{Cu}_{48} \mathrm{Al}_{4}$ & 1.60 \\
\hline $\mathrm{Ca}_{65} \mathrm{Mg}_{8.54} \mathrm{Li}_{9.96} \mathrm{Sn}_{16.5}$ & 1.61 \\
\hline $\mathrm{Ca}_{65} \mathrm{Mg}_{8.31} \mathrm{Li}_{9.69} \mathrm{Sn}_{17}$ & 2.00 \\
\hline $\mathrm{Yb}_{62.5} \mathrm{Mg}_{17.5} \mathrm{Cu}_{5} \mathrm{Zn}_{15}$ & 2.00 \\
\hline $\mathrm{Ce}_{70} \mathrm{Al}_{10} \mathrm{Ni}_{10} \mathrm{Cu}_{10}$ & 1.97 \\
\hline$\left(\mathrm{Ce}_{20} \mathrm{La}_{80}\right)_{68} \mathrm{Al}_{10} \mathrm{Cu}_{20} \mathrm{Co}_{2}$ & 1.89 \\
\hline $\mathrm{Ce}_{68} \mathrm{Al}_{10} \mathrm{Nb}_{2} \mathrm{Cu}_{20}$ & 1.88 \\
\hline$\left(\mathrm{Ce}_{80} \mathrm{La}_{20}\right)_{68} \mathrm{Al}_{10} \mathrm{Cu}_{20} \mathrm{Co}_{2}$ & 1.88 \\
\hline $\mathrm{Ce}_{68} \mathrm{Al}_{10} \mathrm{Co}_{2} \mathrm{Cu}_{20}$ & 1.88 \\
\hline $\mathrm{Ce}_{68} \mathrm{Al}_{10} \mathrm{Ni}_{2} \mathrm{Cu}_{20}$ & 1.87 \\
\hline $\mathrm{La}_{60} \mathrm{Al}_{20} \mathrm{Co}_{20}$ & 1.87 \\
\hline $\mathrm{Dy}_{55} \mathrm{Al}_{25} \mathrm{Co}_{20}$ & 1.85 \\
\hline $\mathrm{Pr}_{55} \mathrm{Al}_{25} \mathrm{CO}_{20}$ & 1.78 \\
\hline $\mathrm{Tb}_{55} \mathrm{Al}_{25} \mathrm{Co}_{20}$ & 1.82 \\
\hline $\mathrm{Ho}_{55} \mathrm{Al}_{25} \mathrm{Co}_{20}$ & 1.78 \\
\hline $\mathrm{Er}_{55} \mathrm{Al}_{25} \mathrm{Co}_{20}$ & 1.76 \\
\hline $\mathrm{Tm}_{55} \mathrm{Al}_{25} \mathrm{Co}_{20}$ & 1.75 \\
\hline $\mathrm{Tm}_{39} \mathrm{Y}_{16} \mathrm{Al}_{25} \mathrm{Co}_{20}$ & 1.75 \\
\hline $\mathrm{Lu}_{39} \mathrm{Y}_{16} \mathrm{Al}_{25} \mathrm{Co}_{20}$ & 1.75 \\
\hline $\mathrm{Lu}_{45} \mathrm{Y}_{10} \mathrm{Al}_{25} \mathrm{Co}_{20}$ & 1.74 \\
\hline $\mathrm{Lu}_{55} \mathrm{Al}_{25} \mathrm{Co}_{20}$ & 1.74 \\
\hline $\mathrm{Mg}_{65} \mathrm{Cu}_{25} \mathrm{Gd}_{10}$ & 1.74 \\
\hline $\mathrm{Mg}_{65} \mathrm{Cu}_{25} \mathrm{Y}_{9} \mathrm{Gd}_{1}$ & 1.71 \\
\hline $\mathrm{Mg}_{65} \mathrm{Cu}_{25} \mathrm{Y}_{10}$ & 1.70 \\
\hline $\mathrm{Mg}_{65} \mathrm{Cu}_{25} \mathrm{Y}_{8} \mathrm{Gd}_{2}$ & 1.70 \\
\hline $\mathrm{Mg}_{65} \mathrm{Cu}_{25} \mathrm{Y}_{5} \mathrm{Gd}_{5}$ & 1.69 \\
\hline $\mathrm{Mg}_{65} \mathrm{Cu}_{25} \mathrm{~Tb}_{10}$ & 1.68 \\
\hline
\end{tabular}

Fig. 1. (Color online) A schematic illustration of radial distribution function (RDF) $g(r)$. 
dependent atomic strain $\gamma_{\text {atomic }}$ measured in in-situ loading scattering tests as illustrated in Fig. 2(a) (For more details, see Supplementary data) calculated with the shift of the peaks of the RDF as illustrated in Fig. 2(b) develops on the range of $0-2 \mathrm{~nm}$ on RDF with increasing external elastic load. The $\gamma_{\text {atomic }}$ is proposed to stem from the anelastic rearrangements between different atoms shells [22] on RDF as shown in Fig. 2(c), which underpin the macroscopic mechanical behavior of amorphous alloys. Based on the concept of shear transformation, the STZ in amorphous solids can thereby be considered from a mean filed perspective as the cooperative rearrangement of these shells of atoms on RDF, i.e., an atomic structure-based STZ (SSTZ). For the isotropic amorphous structure, the SSTZ is of a spherical shape, of which the size can be determined based on the distribution of $\gamma_{\text {atomic }}$ measured by the shift of the peaks of the RDF in the loading direction as shown in Fig. 2(a) and also by the RDF in the transverse direction to external loading [22]. For $\gamma_{\text {atomic }}$ relying on external stress level, the size of SSTZ also depends on the external stress. Based on previously performed X-ray scattering tests [21-23], approaching the yielding point of amorphous alloys, the SSTZ is examined to be of a radius of 1-2 nm as collected in Table 2 matches well the size of $2-3 \mathrm{~nm}$ in diameter of a nowadays recognized STZ [14]. On the other hand, the concept of the activation of the shear transformation event [3] in transition state theory remains the same to that in the reference [10] as shown in Fig. 2(d). The SSTZ can thus be integrated into the flow theory based on STZ [10]: $\left.\dot{\gamma}=\alpha \gamma_{0} v_{G} \operatorname{expl}-\left(\Delta \varepsilon+\gamma_{0} \hat{\tau} \Omega_{f}\right) / k T\right\rfloor \sinh \left(\sigma \gamma_{0} \Omega_{f} / k T\right)$, for the "event" nature of STZs of which the activations are represented in the equation by an normal mode frequency $v_{G}$ multiplied by a stress and temperature dependent probability $\left.\operatorname{expl}-\left(\Delta \varepsilon+\gamma_{0} \hat{\tau} \Omega_{f}\right) / k T\right\rfloor \sinh$ $\left(\sigma \gamma_{0} \Omega_{f} / k T\right)$, where $\dot{\gamma}$ is strain rate; $\gamma_{0}$ is the elementary strain carried by a SSTZ event; $\alpha$ is a numerical coefficient: $\Delta \varepsilon$ is the elastic strain energy; $\hat{\tau}$ is the ideal shear resistance; here $\Omega_{f}$ is the size of SSTZ; $k$ is the
Boltzmann constant; $T$ is temperature; $\sigma$ is flow stress. For detailed derivation of the flow equations, one is referred to the references $[10,11]$.

\section{Discussion}

\subsection{Applicability}

It is noted that the SSTZ is based on the correlation effect between shear transformation events $[31,32]$. Were there no correlation, the case would be simple liquid where thermal kinetic movements of individual atoms would manage the flow of the system. Due to the correlation between shear transformation events, the size and shape of the SSTZ can be determined from the mean perspective by the distribution of the length scale dependent atomic strain $\gamma_{\text {atomic }}$ as shown in Fig. 2(a), because the atom packing shown in the RDF is both spatially and temporally averaged over the whole deformed system. Hence, correlation effect is the basis of the SSTZ and determines that the application of the SSTZ is confined to the field where correlation exists, like in supercooled liquids (especially near the glass transition temperature $T_{g}$ ), glasses, colloids and near jammed granular materials [33]. For example, the flow of supercooled metallic liquids can be explained with the SSTZ as shown in Fig. 3. At lower flow stress level, the cooperative rearrangement of the nearest neighbors would be enough to afford the flow rate. Consequently, the size of SSTZ is small and the flow is both microscopically and macroscopically homogeneous, i.e., Newtonian mode flow with the viscosity being a constant. With increasing flow stress level at higher flow rates, cooperative rearrangements of more shells of atoms are involved in flow. The SSTZ becomes larger and the length scale dependent atomic strain $\gamma_{\text {atomic }}$ exhibits fluctuation (See Fig. 2(a)). For the expansion and contraction of different atom shells indicated by the fluctuation of $\gamma_{\text {atomic }}$, shear dilation takes place and induces shear thinning, i.e., a

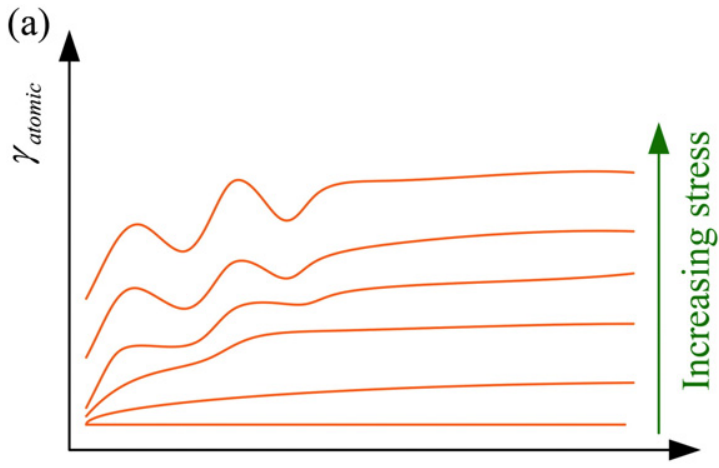

Distance from average atom, $r$ (b)

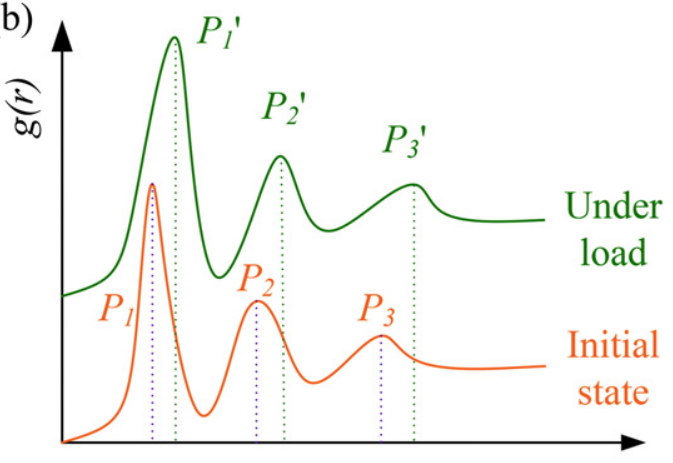

Distance from average atom, $r$
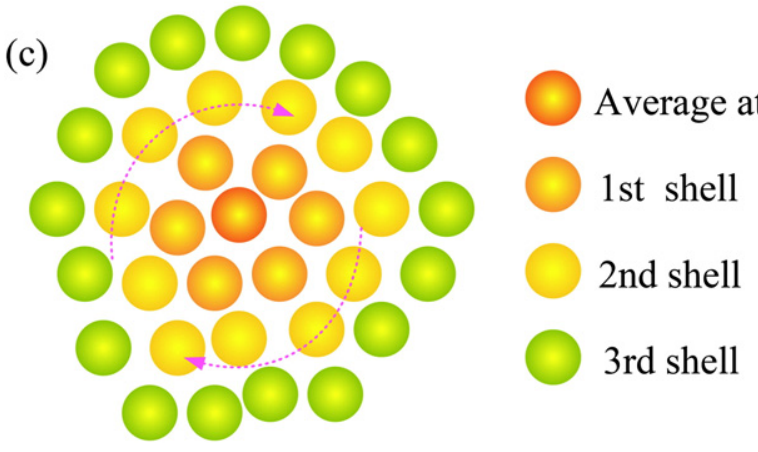

(d)

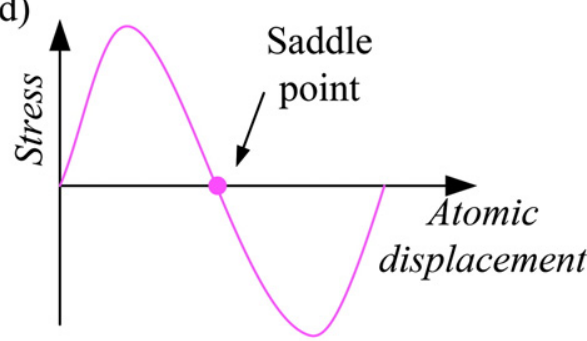

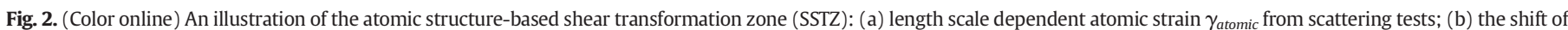

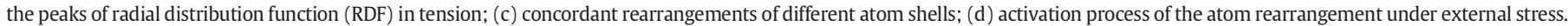

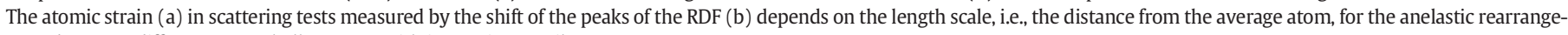
ment between different atom shells on RDF with increasing tensile stress. 
Table 2

The size of the atomic structure-based shear transformation zone (SSTZ) measured in scattering tests at certain stress levels in tension.

\begin{tabular}{llll}
\hline Composition & Stress(GPa) & $\begin{array}{l}\text { SSTZ radii } \\
(\mathrm{nm})\end{array}$ & $\begin{array}{l}\text { Yielding strength } \\
(\mathrm{GPa})\end{array}$ \\
\hline $\mathrm{Cu}_{46} \mathrm{Zr}_{46} \mathrm{Al}_{8}$ (Ref. [21]) & 1.75 & 1.3 & 1.9 \\
$\mathrm{Zr}_{48} \mathrm{Cu}_{43} \mathrm{Al}_{7} \mathrm{Ag}_{2}$ (Ref. [21]) & 1.5 & 1.5 & 1.6 \\
$\mathrm{Zr}_{62} \mathrm{Al}_{8} \mathrm{Ni}_{13} \mathrm{Cu}_{17}$ (Ref. [21]) & 1.4 & 1.5 & 1.6 \\
$\mathrm{La}_{62} \mathrm{Al}_{14}\left(\mathrm{Cu}_{5 / 6} \mathrm{Ag}_{1 / 6}\right)_{14} \mathrm{Co}_{5} \mathrm{Ni}_{5}$ & 0.5 & 1.6 & 0.6 \\
$\quad\left(\mathrm{Ref}^{[21])}\right.$ & & & \\
$\mathrm{Zr}_{57} \mathrm{Ti}_{5} \mathrm{Ni}_{8} \mathrm{Al}_{10} \mathrm{Cu}_{20}$ (Ref. [22]) & 1.08 & 1.8 & 1.8 \\
$\mathrm{Zr}_{46.5} \mathrm{Cu}_{45} \mathrm{Al}_{7} \mathrm{Ti}_{1.5}$ (Ref. [23]) & 1.38 & 1.5 & 1.6 \\
\hline
\end{tabular}

decreased apparent viscosity [34]. As the correlation increases with flow stress at sufficiently high flow rates, jamming occurs and the fracture of supercooled liquid sets in before flow starts. All these phenomena have been reported in the rheology of amorphous alloys [1,35].

Moreover, the SSTZ can be understood as an extrapolation of the Gibbs ensemble, except that the Gibbs ensembles deal with thermal equilibrium systems and here we are using the SSTZ to tackle the deformation of amorphous solids which is usually far from equilibrium on mean field assumption. From this aspect of view, it is important to note that a local configuration rearrangement of an atomic cluster of several hundreds of atoms in the amorphous system is usually trivial to a macroscopic system of an Avogadro constant number of atoms and hardly affects the macroscopic behavior of such a system of statistical character (i.e., containing enough large number of atoms). Only in the sense of mean field, percolated many of these clusters would determine the state of the system [36]. This is the physical meaning of the SSTZ, i.e., to give a structure basis of the local shear transformation in terms of RDF from the mean perspective. In turn, The SSTZ also supports the proposition that the structural origin of the properties of amorphous solids mainly lies in the short to medium range of 1-3 $\mathrm{nm}$ [37].

\subsection{Application}

It has been reported that the yielding behavior of amorphous alloys can be universalized with a power law criterion both in the sense of mechanical yielding [30] and in the sense of stress driven glass transition [38]. The energy required to overcome the barrier of yielding and glass transition mainly depends on the structural characteristic of STZ and also the interaction potentials between the constituent atoms [29]. The power-law scaling of the atom packing in amorphous alloys extracted based on the first peak position of the structure factor has been revealed to suggest the self-similarity of their amorphous structure on medium range $0.5-1.5 \mathrm{~nm}$ [19], exactly the size of the SSTZ. The similar distributions of length scale dependent atomic strain $\gamma_{\text {atomic }}$ of several amorphous alloys $[21,23]$ near macroscopic yielding are also shown in
Fig. 4(a) to support the self-similarity of their structures. Therefore, taking the glass transition temperature $T_{g}$ to represent the potentials between the constituent atoms of different systems of amorphous alloys, for the self-similar medium range structure of amorphous alloys, the energy barrier for yielding averaged over the volume of the SSTZ (i.e., the energy barrier density) should depend linearly on $T_{g}$. This is consistent with the proposed universal yielding criterion, where near linear relevance between the energy barrier density [30]: $W / \Omega=\left(8 / \pi^{2}\right) \gamma_{C}^{2} S G \approx 2.4 \gamma_{C}^{2} G$, (W is the energy barrier; $\Omega$ is the volume of STZ; $\varsigma$ is a coefficient; $\gamma_{C}$ is the critical shear strain; $G$ is the shear modulus) and the glass transition temperature $T_{g}$ of different amorphous alloys [3] is plotted in Fig. 4(b) (For more details, see Table SI in Supplementary data). The deviation of the data points from the linear relation surrounded by a dotted ellipse which represent the Ni-based or iron-based amorphous alloys probably originates from the determination of $T_{g}$ of these systems for its high temperature value and the vague glass transition region on their thermal analysis traces. Hence, the SSTZ will provide the empirical universal yielding criterion of amorphous alloys with a structure basis.

One key feature of the deformation of amorphous solids is shear dilation [39]. As the outer shell of the SSTZ exhibits larger strain in Fig. 2(a), the volume of inner shells expands for the geometrical compatibility of deformation. This is the structural origin of shear dilation. In terms of the SSTZ, shear dilation can be quantified as the relative shift of the peak of RDF or structure factor and compared to the relaxation enthalpy, atomic level stress [40] and other measures of free volume concentration [41]. To demonstrate this proposition, we have calculated the fraction of anelastic deformation which is closely related to shear dilation. As shown in Fig. 5, based on the length scale dependent atomic strain [22] $\gamma_{\text {atomic }}$ induced by anelastic deformation between $r_{i}$ and $r_{e}$, the fraction of anelastic deformation (the area between the solid line and the dashed line indicated by the arrow) is estimated to be $10-20 \%$ of the total elastic strain (the area of the rectangle $a r_{i} r_{e} b$ ) at a stress level of $0.5 \sim 0.9 \sigma_{y}$ ( $\sigma_{y}$ is the yielding strength), matching previous researches very well [42] (for more details, see Supplementary data). Therefore, the SSTZ will also provide the anelastic deformation and shear dilation of amorphous alloys with a structure basis.

Poisson ratio and fragility have been reported to correlate with the dynamic heterogeneity of amorphous solids in terms of the shear-like heterogeneity and the density heterogeneity [43], as lower Poisson ratio and lower fragility value (i.e., strong glass) suggesting higher proportion of density fluctuation. The shift of the peaks of the RDF would reflect the density heterogeneity and the relative shift of the peaks would indicate the shear-like heterogeneity on different length scales, as shown in Fig. 2(a) the fluctuation of the length scale dependent atomic strain $\gamma_{\text {atomic }}$ exhibiting the contraction and expansion of different atoms shells and the shear-like heterogeneity between the atom shells. This could probably be why $\mathrm{La}_{62} \mathrm{Al}_{14}\left(\mathrm{Cu}_{1 / 6} \mathrm{Ag}_{1 / 5}\right){ }_{14} \mathrm{Ni}_{5} \mathrm{CO}_{5}$ alloy of a lower Poisson ratio of (0.33-0.36) [27] exhibits larger fluctuation

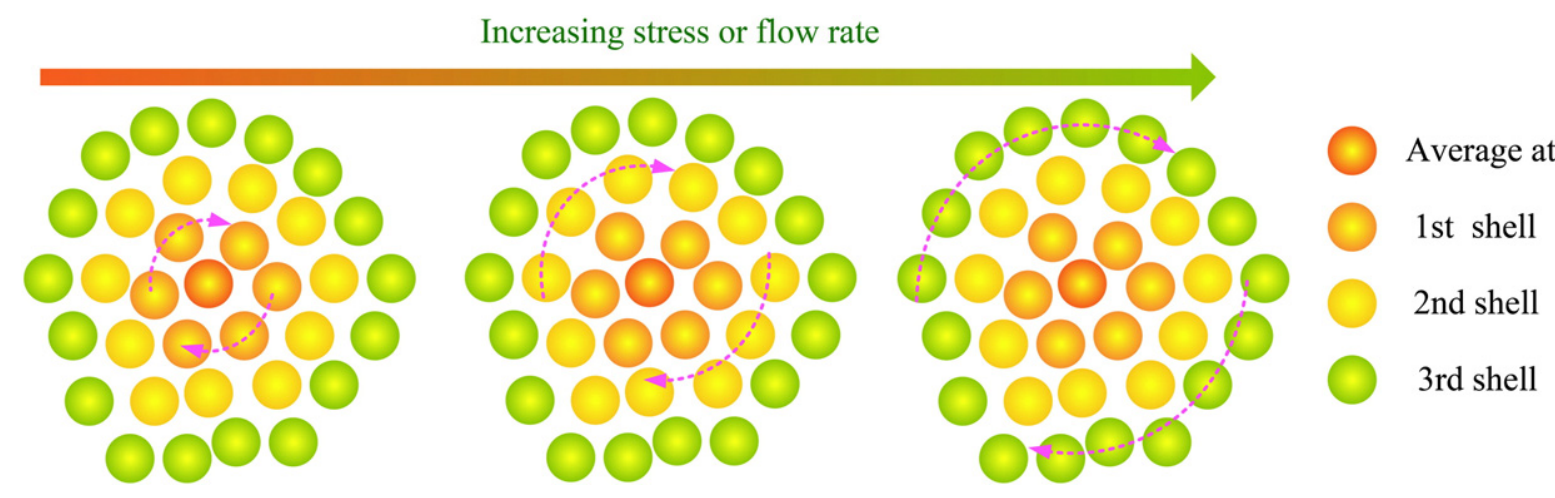

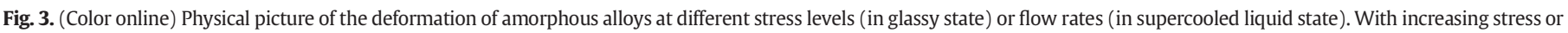
flow rate, more shells of atoms will be involved in the cooperative rearrangement, exhibiting an increasing size of the atomic structure-based shear transformation zone (SSTZ). 

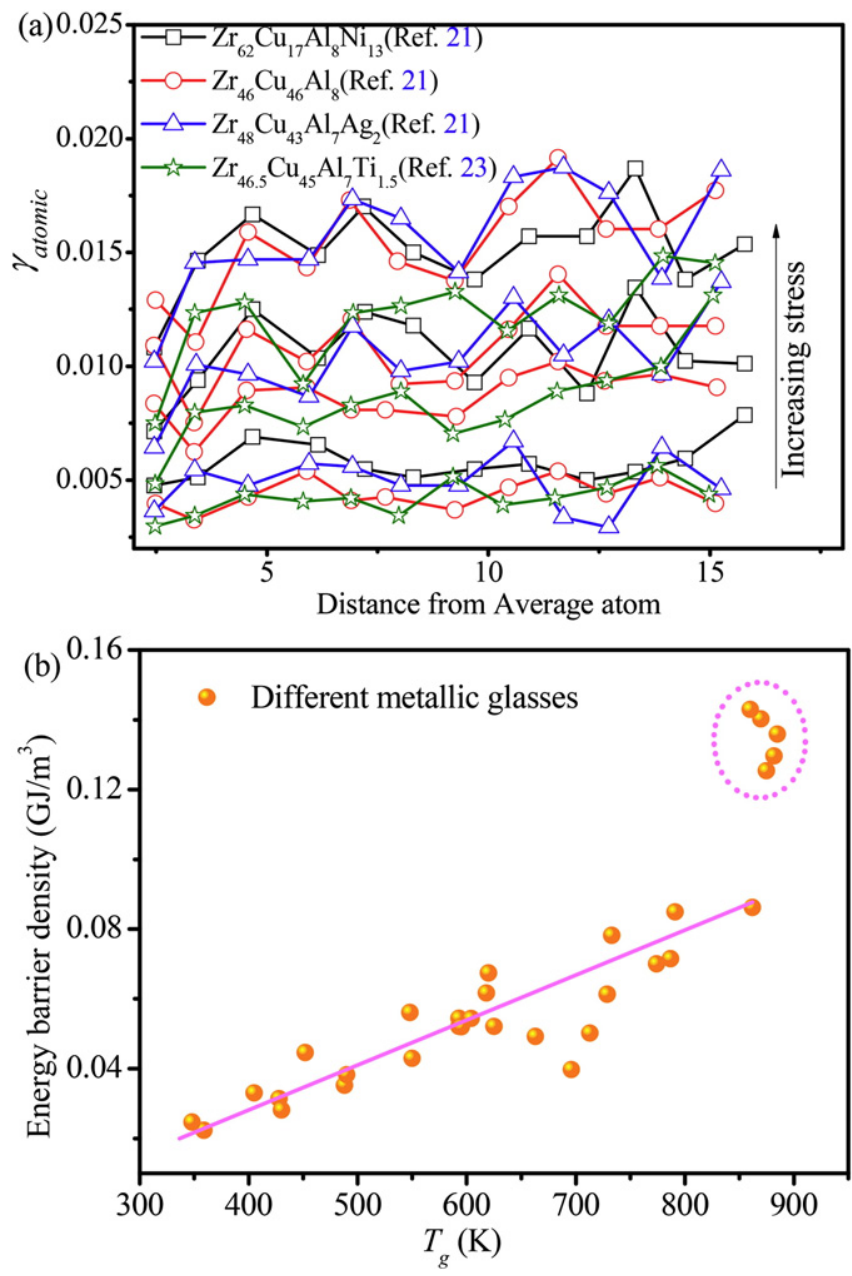

Fig. 4. (Color online) Length scaled dependent atomic strain $\gamma_{\text {atomic }}$ of several amorphous alloys from the Refs. [21,23] (a); Near linear correlation between the energy barrier density of the shear transformation zone (STZ) and the glass transition temperature of different amorphous alloys from Ref. [3], the solid line is an eye-guide (b).

[21] of $\gamma_{\text {atomic }}$ than $\mathrm{Zr}_{62} \mathrm{Cu}_{17} \mathrm{~A}_{18} \mathrm{Ni}_{13}$ alloy of a higher Poisson ratio of (0.37-0.38) [27]. Along this approach, the fragility of amorphous alloys can also be understood with a structure background, for the intimate correlation between fragility and Poisson ratio [44,45].

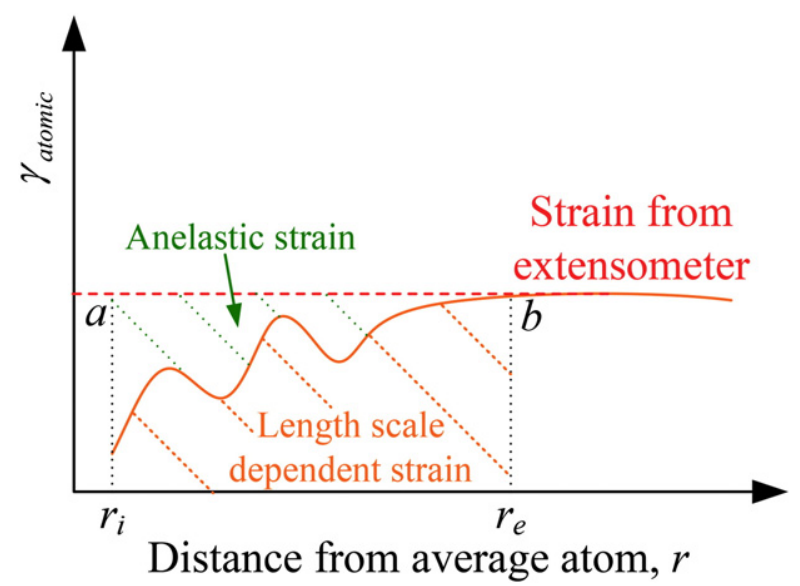

Fig. 5. (Color online) Calculation of the fraction of anelastic strain based on the length scale dependent atomic strain $\gamma_{\text {atomic }}$.

\section{Conclusion}

In summary, an atomic structure-based shear transformation zone (SSTZ) of amorphous solids based on radial distribution function (RDF) is proposed without modifying current flow theory to bridge STZ to the atomic structure and to protrude its "event" nature. Detailed aspects, for instance, the size and shape, of the SSTZ can be resolved on the RDF in the in-situ loading scattering tests. More importantly, the SSTZ provides both the universal yielding criterion and the anelastic deformation of amorphous alloys with a structure basis. Future works focusing on the SSTZ to uncover the mechanical properties and underlying physics of amorphous solids are expected, because the SSTZ is of specific structural characteristic and presents an accessible approach to explore the structure-property relationship in the mechanical behavior of amorphous solids.

\section{Acknowledgments}

This work was financially supported by the National Nature and Science Foundation of China under Grant No. 11472287, the National Basic Research Program of China under Grant No. 2012CB937500, and the CAS/SAFEA International Partnership Program for Creative Research Teams.

\section{Appendix A. Supplementary data}

Supplementary data to this article can be found online at http://dx. doi.org/10.1016/j.jnoncrysol.2014.12.020.

\section{References}

[1] C.A. Schuh, T. Hufnagel, U. Ramamurty, Mechanical behavior of amorphous alloys, Acta Mater. 55 (2007) 4067-4109.

[2] A.L. Greer, Y.Q. Cheng, E. Ma, Shear bands in metallic glasses, Mater. Sci. Eng. R. Rep. 74 (2013) 71-132.

[3] M.W. Chen, Mechanical behavior of metallic glasses: microscopic understanding of strength and ductility, Annu. Rev. Mater. Res., Annu. Rev. Annual Reviews, Palo Alto, 2008, pp. 445-469.

[4] G.I. Taylor, The mechanism of plastic deformation of crystals. Part I. Theoretical, Proc. R. Soc. Lond. A 145 (1934) 362-387.

[5] E. Orowan, Z. Phys. 89 (1934) (605-613; 614-633; 634-659).

[6] M. Polani, Z. Phys. 89 (1934) 660-664.

[7] R. Peierls, The size of a dislocation, Proc. Phys. Soc. 52 (1940) 34-37.

[8] F.R.N. Nabarro, Dislocations in a simple cubic lattice, Proc. Phys. Soc. 59 (1947) 256-272.

[9] J.D. Eshelby, Uniformly moving dislocations, Proc. Phys. Soc. A 62 (1949) 307-314.

[10] A.S. Argon, Plastic deformation in metallic glasses, Acta Metall. 27 (1979) 47-58.

[11] M.L. Falk, J.S. Langer, Dynamics of viscoplastic deformation in amorphous solids, Phys. Rev. E. 57 (1998) 7192-7205.

[12] H.B. Yu, W.H. Wang, H.Y. Bai, Y. Wu, M.W. Chen, Relating activation of shear transformation zones to beta relaxations in metallic glasses, Phys. Rev. B 81 (2010) 220201.

[13] J.C. Ye, J. Lu, C.T. Liu, Q. Wang, Y. Yang, Atomistic free-volume zones and inelastic deformation of metallic glasses, Nat. Mater. 9 (2010) 619-623.

[14] D. Pan, A. Inoue, T. Sakurai, M.W. Chen, Experimental characterization of shear transformation zones for plastic flow of bulk metallic glasses, Proc. Natl. Acad. Sci. U. S. A. 105 (2008) 14769-14772.

[15] H.L. Peng, M.Z. Li, B.A. Sun, W.H. Wang, Characterization of mechanical heterogeneity in amorphous solids, J. Appl. Phys. 112 (2012) 023516.

[16] H. Rosner, M. Peterlechner, C. Kubel, V. Schmidt, G. Wilde, Density changes in shear bands of a metallic glass determined by correlative analytical transmission electron microscopy, Ultramicroscopy 142 (2014) 1-9.

[17] J. Bunz, T. Brink, K. Tsuchiya, F.Q. Meng, G. Wilde, K. Albe, Low temperature heat capacity of a severely deformed metallic glass, Phys. Rev. Lett. 112 (2014) 135501.

[18] H.F. Poulsen, J.A. Wert, J. Neuefeind, V. Honkimaki, M. Daymond, Measuring strain distributions in amorphous materials, Nat. Mater. 4 (2005) 33-36.

[19] D. Ma, A.D. Stoica, X.L. Wang, Power-law scaling and fractal nature of medium-range order in metallic glasses, Nat. Mater. 8 (2009) 30-34.

[20] T.C. Hufnagel, R.T. Ott, J. Almer, Structural aspects of elastic deformation of a metallic glass, Phys. Rev. B 73 (2006) 064204.

[21] X.D. Wang, J. Bednarcik, H. Franz, H.B. Lou, Z.H. He, Q.P. Cao, J.Z. Jiang, Local strain behavior of bulk metallic glasses under tension studied by in situ X-ray diffraction, Appl. Phys. Lett. 94 (2009) 011911.

[22] U.K. Vempati, P.K. Valavala, M.L. Falk, J. Almer, T.C. Hufnagel, Length-scale dependence of elastic strain from scattering measurements in metallic glasses, Phys. Rev. B 85 (2012) 214201. 
[23] L.Y. Chen, B.Z. Li, X.D. Wang, F. Jiang, Y. Ren, P.K. Liaw, J.Z. Jiang, Atomic-scale mechanisms of tension-compression asymmetry in a metallic glass, Acta Mater. 61 (2013) 1843-1850.

[24] A.S. Argon, H.Y. Kuo, Plastic flow in a disordered bubble raft (an analog of a metallic glass), Mater. Sci. Eng. 39 (1979) 101-109.

[25] P. Schall, D.A. Weitz, F. Spaepen, Structural rearrangements that govern flow in colloidal glasses, Science 318 (2007) 1895-1899.

[26] M. Zaiser, P. Hahner, Oscillatory modes of plastic deformation: theoretical concepts, Phys. Status Solidi B 199 (1997) 267-330.

[27] W.H. Wang, The elastic properties, elastic models and elastic perspectives of metallic glasses, Prog. Mater. Sci. 57 (2012) 487-656.

[28] Y.Q. Cheng, E. Ma, Atomic-level structure and structure-property relationship in metallic glasses, Prog. Mater. Sci. 56 (2011) 379-473.

[29] S.G. Mayr, Activation energy of shear transformation zones: a key for understanding rheology of glasses and liquids, Phys. Rev. Lett. 97 (2006) 195501.

[30] W.L. Johnson, K. Samwer, A universal criterion for plastic yielding of metallic glasses with a $\left(T / T_{g}\right)^{2 / 3}$ temperature dependence, Phys. Rev. Lett. 95 (2005) 195501.

[31] M. Zhang, L. Liu, Y. Wu, Facilitation and correlation of flow in metallic supercooled liquid, J. Chem. Phys. 139 (2013) 164508.

[32] A. Nicolas, J. Rottler, J.L. Barrat, Spatiotemporal correlations between plastic events in the shear flow of athermal amorphous solids, Eur. Phys. J. E Soft Matter 37 (2014) 50

[33] D. Chandler, J.P. Garrahan, Dynamics on the way to forming glass: bubbles in spacetime, Annu. Rev. Phys. Chem. 61 (2010) 191-217.

[34] A. Lemaître, Rearrangements and dilatancy for sheared dense materials, Phys. Rev. Lett. 89 (2002) 195503.
[35] J. Lu, G. Ravichandran, W.L. Johnson, Deformation behavior of the Zr41.2Ti13.8Cu12.5Ni10Be22.5 bulk metallic glass over a wide range of strainrates and temperatures, Acta Mater. 51 (2003) 3429-3443.

[36] J.S. Harmon, M.D. Demetriou, W.L. Johnson, K. Samwer, Anelastic to plastic transition in metallic glass-forming liquids, Phys. Rev. Lett. 99 (2007) 135502.

[37] G. Baldi, M. Zanatta, E. Gilioli, V. Milman, K. Refson, B. Wehinger, B. Winkler, A. Fontana, G. Monaco, Emergence of crystal-like atomic dynamics in glasses at the nanometer scale, Phys. Rev. Lett. 110 (2013) 185503.

[38] Y.H. Liu, C.T. Liu, W.H. Wang, A. Inoue, T. Sakurai, M.W. Chen, Thermodynamic origins of shear band formation and the universal scaling law of metallic glass strength, Phys. Rev. Lett. 103 (2009) 065504.

[39] F. Spaepen, A microscopic mechanism for steady state inhomogeneous flow in metallic glasses, Acta Metall. 25 (1977) 407-415.

[40] T. Egami, Atomic level stresses, Prog. Mater. Sci. 56 (2011) 637-653.

[41] A. Slipenyuk, J. Eckert, Correlation between enthalpy change and free volume reduction during structural relaxation of Zr55Cu30Al10Ni5 metallic glass, Scr. Mater. 50 (2004) 39-44.

[42] W. Dmowski, T. Iwashita, C.P. Chuang, J. Almer, T. Egami, Elastic heterogeneity in metallic glasses, Phys. Rev. Lett. 105 (2010) 205502.

[43] E. Duval, T. Deschamps, L. Saviot, Poisson ratio and excess low-frequency vibrational states in glasses, J. Chem. Phys. 139 (2013) 064506

[44] M.O. Jiang, L.H. Dai, Intrinsic correlation between fragility and bulk modulus in metallic glasses, Phys. Rev. B 76 (2007) 054204.

[45] V.N. Novikov, A.P. Sokolov, Poisson's ratio and the fragility of glass-forming liquids, Nature 431 (2004) 961-963. 